INPLASY

PROTOCOL

To cite: Gandia et al. Strategies

to assess and promote university students' socioemotional competencies in socio-educational and health fields: a scoping review. Inplasy protocol 202120076. doi:

10.37766/inplasy2021.2.0076

Received: 23 February 2021

Published: 23 February 2021

Corresponding author: Natalia Gandia Carbonell

natalia.gandia@e-campus.uab.cat

Author Affiliation:

Autonomous University of Barcelona

Support: MICINN: PGC2018-100675BI00.

Review Stage at time of this submission: Piloting of the study selection process.

Conflicts of interest:

None declared.

\section{Strategies to assess and promote university students' socio-emotional competencies in socio-educational and health fields: a scoping review}

Gandia, N1; Losilla, JM2; Viguer, $\mathrm{P}^{3}$.

Review question / Objective: What strategies have been proposed to assess or promote university students' socioemotional competencies in socio-educational and health fields?

Condition being studied: Strategies to assess and promote socio-emotional competencies in university education at all levels in health and socio-educational fields. Professionals trained in these fields need an adequate level of socioemotional competence in order to effectively attend to the individual needs, well-being and quality of life of service endusers.

Information sources: The scoping review will be performed using PubMed, US National Library of Medicine, by National Center for Biotechnology Information (NCBI); Education Resources Information Center (ERIC) by ProQuest; PsycINFO, Psychological Information, by APA PsycNET; Scopus by Elsevier; as well as Dissertations \& Theses Global by ProQuest and the first 50 Google search results for grey literature. Search alerts will be set in all the databases. The search strategy will follow the Peer Review of Electronic Search Strategies (PRESS) guidelines (McGowan et al., 2016).

INPLASY registration number: This protocol was registered with the International Platform of Registered Systematic Review and Meta-Analysis Protocols (INPLASY) on 23 February 2021 and was last updated on 23 February 2021 (registration number INPLASY202120076).

\section{INTRODUCTION}

Review question / Objective: What strategies have been proposed to assess or promote university students' socioemotional competencies in socioeducational and health fields?
Rationale: Various theoretical models have addressed the emotional intelligence construct, such as the ability model (Salovey and Mayer, 1990), the mixed model (Bar-On, 1997; Goleman, 1995) and the trait model (Petrides \& Furnham, 2001). In this 
work, we will use the concept of socioemotional competence, defined by Bar-On (2006) as a set of knowledge, capacities, skills and attitudes for understanding, expressing and appropriately regulating one's own and others' emotional phenomena and coping with the demands of one's environment. We will focus on the socio-emotional competencies of university students in health and socioeducational fields, who may experience work situations characterized by complexity, vulnerability and conflict (Rosa et al., 2015), which demand adequate selfknowledge and emotional management, since poor personal management of workcare situations can lead to personal distress affecting the quality of care and, among other aspects, the component of empathy in professional practice (Thomas et al., 2007). Promoting the development of socio-emotional competencies in university education can lead to personal and professional benefits, such as better stress management (Carvalho et al., 2018), greater effectiveness in verbal and non-verbal communications (Carvalho et al., 2018; Holston \& Taylor, 2016), increased capacity for conflict management (Cleary et al., 2018), facilitation of the building of therapeutic relationships (Augusto-Landa \& Montes-Berges, 2009), as well as better teamwork skills and the development of more effective interactions between professionals, better decision-making and development of critical thinking (Barkhordari \& Rostambeygi, 2013).

Condition being studied: Strategies to assess and promote socio-emotional competencies in university education at all levels in health and socio-educational fields. Professionals trained in these fields need an adequate level of socio-emotional competence in order to effectively attend to the individual needs, well-being and quality of life of service end-users.

\section{METHODS}

Search strategy: The search strategy will consist of two groups of search terms: ("emotional skills" OR "socioemotional skills" OR "socio-emotional skills" OR "emotional competences" OR "socioemotional competences" OR "socioemotional competences" OR "emotional abilities" OR "socioemotional abilities" OR "socio-emotional abilities" OR "emotional intelligence" OR "socioemotional intelligence" OR "socio-emotional intelligence" OR "emotional education" OR "socioemotional education" OR "socioemotional education" OR "emotional learning" OR "socioemotional learning" OR "socio-emotional learning"); AND ("undergraduat*" OR "postgraduat"” OR "graduat *" OR "universit*" OR "postsecondary" OR "college" OR "higher education" OR "tertiary education" OR "bachelor*"). This search strategy will be adapted to the syntax and specific characteristics of each bibliographic database. The systematic search will be performed in February 2021 and limited by methodology (empirical studies), age (18 years and older) and language (English, Spanish and Catalan).

Participant or population: University students at all levels in socio-educational and health fields.

Intervention: Procedures for assessing university students' socio-emotional competencies in health and socioeducational fields. Interventions promoting university students' socio-emotional competencies in health and socioeducational fields.

\section{Comparator: None.}

Study designs to be included: Empirical studies with experimental (randomized and non-randomized), non-experimental (cohort, case-control and cross-sectional), single-case and qualitative designs.

Eligibility criteria: Eligible are empirical studies on assessment and intervention measures regarding socio-emotional competencies in undergraduate, graduate and postgraduate university students being trained as professionals in socioeducational and health fields with a view to effectively attending to the individual needs, well-being and quality of life of 
service end-users. Theoretical and case report studies will be excluded and also studies whose objective is exclusively the academic review or evaluation of university study plans in the aforementioned areas.

Information sources: The scoping review will be performed using PubMed, US National Library of Medicine, by National Center for Biotechnology Information (NCBI); Education Resources Information Center (ERIC) by ProQuest; PsycINFO, Psychological Information, by APA PsycNET; Scopus by Elsevier; as well as Dissertations \& Theses Global by ProQuest and the first 50 Google search results for grey literature. Search alerts will be set in all the databases. The search strategy will follow the Peer Review of Electronic Search Strategies (PRESS) guidelines (McGowan et al., 2016).

Main outcome(s): Identification and description of the main characteristics of procedures to assess university students' socio-emotional competencies: theoretical framework, assessed socio-emotional components, form of administration, feasibility, and quality evidence (reliability and validity). Identification and description of the main characteristics of intervention strategies promoting university students' socio-emotional competencies: field (socio-educational and/or health), educational level (degree and/or postgraduate), theoretical framework, promoted socio-emotional components, intervention procedure (e.g., in or out of the classroom, face-to-face or online, simulated or real-life experience, individual or group), evaluation measures of socioemotional competencies and results (e.g., efficacy, effectiveness, efficiency and costs).

Data management: References identified by the search strategy will be entered into Mendeley bibliographic software, and duplicates will be removed. Titles and abstracts will be screened independently by two reviewers. When decisions cannot be made from title and abstract alone, the full paper will be retrieved. Full-text inclusion criteria will be screened independently by two reviewers. Discrepancies during the process will be resolved through discussion (with a third reviewer when necessary). Extracted information will include: general information (author, year); study design (experimental, quasi-experimental, observational, single-case, qualitative); characteristics of the study sample (size, average age, gender distribution, country); characteristics of the measures to assess socio-emotional competence (name or title, theoretical framework, assessed socioemotional components, form of administration, feasibility and quality evidence: reliability and validity); characteristics of the intervention (field: socio-educational or health, educational level: undergraduate, graduate and/or postgraduate, theoretical framework, promoted socio-emotional components, intervention procedure, measurement of socio-emotional competencies, and results: efficacy, effectiveness, efficiency and costs).

Quality assessment / Risk of bias analysis: To maximize the reporting quality of the scoping review, we will follow the PRISMA Extension for Scoping Reviews (PRISMAScR) recommendations (Tricco et al., 2018). The following tools are also expected to be used in order to assess quality and bias risk: - The COSMIN methodology (Mokkink et al., 2018) for evaluating the quality of assessment tools of socio-emotional competencies. - The Cochrane Collaborations's tool (Higgins et al., 2011) for assessing the bias risk of experimental and quasi-experimental studies. - The ROBS tool (Losilla et al., 2018) for assessing the bias risk of observational studies (cohorts, case-control and crosssectional studies). - The checklist proposed by Maggin et al. (2013), based on the evidence standards developed by the What Works Clearinghouse (WWC, Kratochwill et al., 2010) in the evaluation of single-subject research, for assessing the bias risk of single-case studies. - The tool proposed by the National Institute for Health and Care Excellence (NICE, 2012) for assessing the methodological quality of qualitative studies. 
Strategy of data synthesis: We will provide a narrative synthesis of the characteristics and results of the included studies. We will provide a detailed descriptive analysis of the assessment measures of socioemotional competencies and the interventions carried out to promote them.

Subgroup analysis: The identified assessment strategies will be classified and compared according to their theoretical framework, and socioemotional components. The identified intervention strategies will be classified and compared according to their field (socio-educational and/or health), educational level (undergraduate, graduate and/or postgraduate), promoted socioemotional components, and intervention procedure (e.g., in or out of the classroom, face-to-face or online, simulated or real-life experience, individual or group).

Sensitivity analysis: The assessment strategies will be compared according to their feasibility and quality of evidence (reliability and validity). The intervention strategies will be compared according to the year of data collection, research design and bias risk of primary studies.

Language: English.

Country(ies) involved: Spain.

Keywords: Scoping review, socioemotional competencies, university education, university health and care studies, university socio-educational work studies.

Dissemination plans: The results will be disseminated by publication in a peerreviewed journal and presented at relevant conferences.

Contributions of each author:

Author 1 - Natalia Gandia Carbonell - The author will actively participate in all phases of the preparation and writing of the manuscript.

Email: natalia.gandia@e-campus.uab.cat

Author 2 - Josep-Maria Losilla Vidal - The author will actively participate in all phases of the preparation and writing of the manuscript.

Email: josepmaria.losilla@uab.cat

Author 3 - Paz Viguer Seguí - The author will actively participate in all phases of the preparation and writing of the manuscript.

Email: paz.viguer@uv.es 